\title{
O̊GUM TIROL trifft 23. Radiologie Update
}

Am 18.06.2015 fand in der Universitätsklinik Innsbruck ein Fortbildungsabend der Universitätsklinik für Radiologie mit der ÖGUM Tirol in einem spannenden Bogenschluss statt, wobei auf einen möglichst interdisziplinären Austausch Wert gelegt wurde. Gemeinsam mit dem „Radiologie update Innsbruck“, welches eine etablierte radiologische Fortbildung darstellt und zum 32. Mal stattfindend, organisiert von Frau Prof. Gudrun Feuchnter, Priv. Doz. Hannes Gruber und Herrn Prof. Werner Jaschke, wurde ein reger interdiszplinärer Austausch zwischen Klinikern und Radiologen ermöglicht.

Vertreter aus verschiedenen ÖGUM-Arbeitskreisen sprachen zum Thema: „Auf dem Olymp des CEUS : Die Kontrastmittelsonografie jenseits der Basics“.

Dr. Kurt Somavilla, ÖGUM-Arbeitskreisleiter „Allgemeinmedizin“, Fulpmes, begann nach Grußworten der Präsidentin Doz. Dr. Andrea Klauser mit anschaulichen Beispielen aus der Praxis mit: „Was erwartet sich der Praktiker von der Klinik?"

Dieser Vortrag wurde gefolgt von „Intraoperative Kontrastmittelsonografie der Leber", eindrucksvoll wiedergegeben von OA. Dr. Thomas Ybinger, ÖGUM-Arbeits-

kreisleiter „Abdomen“, Kaiser-Franz-Josef-Spital, Wien, wo eine hervorragende Kooperation zwischen Radiologie und Chirurgie ersichtlich wurde.

Im nächsten Vortrag ging OA. Dr. Alexander Loizides, ÖGUM-Arbeitskreisstv. „Bewegungsapparat", Universitätsklinik für Radiologie, Innsbruck, auf „Kontrastmittelsonografie in der Diagnostik von Weichteiltumoren - Ein hilfreiches Tool?" ein

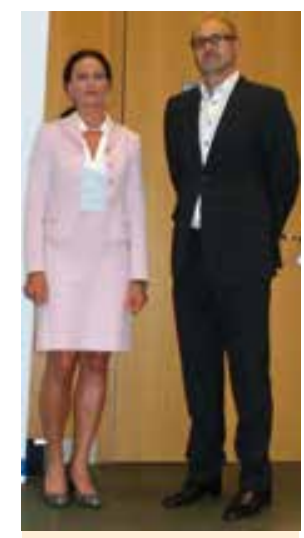

Begrüßung durch die ÖGUM-Präsidentin Univ. Doz. Dr. Klauser und Herrn Richtler (Bracco Österreich)

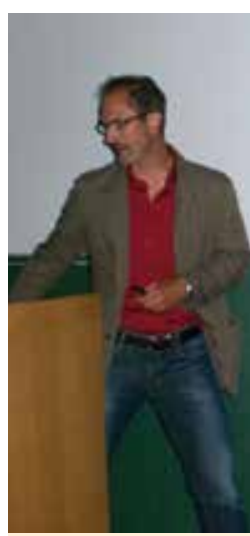

Priv. Doz.

Dr. Hannes Gruber dynamisch in Aktion.... 


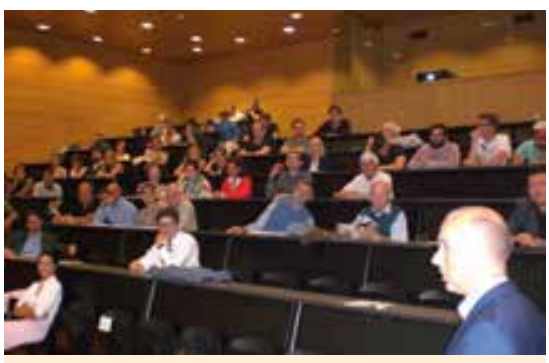

Ein gute gefüllter Hörsaal mit reger Diskussion....

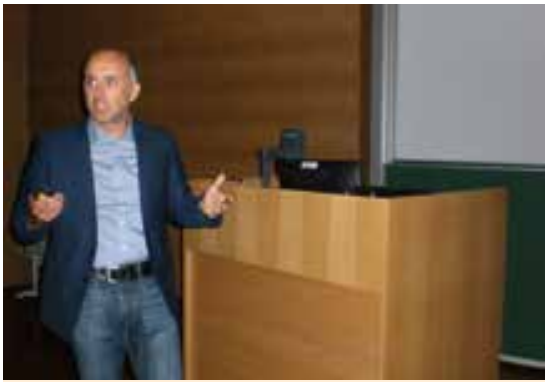

OA. Dr. Sturm diskutiert die Wertigkeit der verschiedenen Sonotechniken.

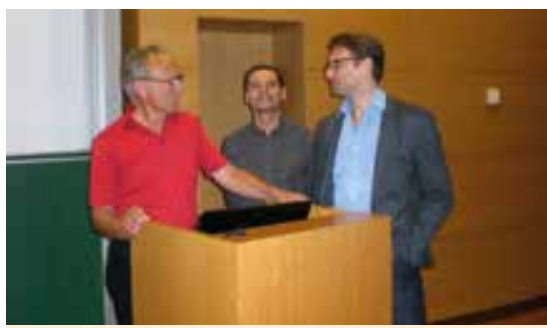

Dr. Somavilla, Priv. Doz. Dr. Aigner und OA. Dr. Ybinger beim Fachsimpeln.

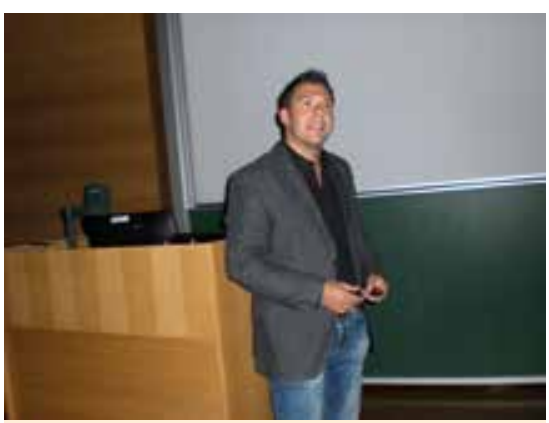

OA. Dr. Loizides bringt Neues bei Weichteil tumoren.

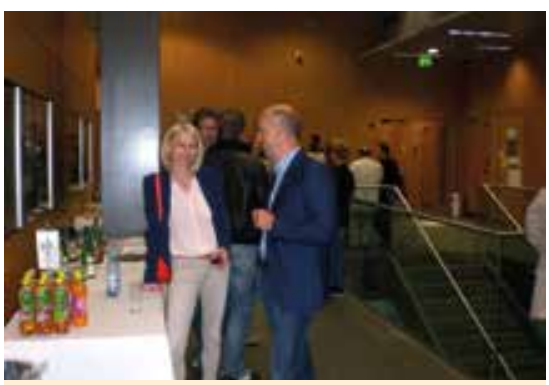

Geselliger Ausklang. und stellte spannende vorläufige weitere Ergebnisse dieser Thematik vor.

Der Kontrastmitteleinsatz wurde kritisch beleutet in „Was erwartetet sich der Kliniker in der Gefäßdiagnostik?“ von OA. Dr. Wolfgang Sturm, ÖGUM-Arbeitskreisleiter „Gefäße“, Universitätsklinik für Innere Medizin, Innsbruck, wobei abschließend Perspektiven zur Diskussion gestellt wurden. Davon gefolgt gab Priv. Doz. Dr. Hannes Gruber, Universitätsklinik für Radiologie, Innsbruck, in seinem Vortrag über „Kontrastmittelsonografie in der Diagnostik von Pathologien der Aorta" einen hochinteressanten Einblick in den Stellenwert dieser Methode.

Abschliessend wurde von Priv. Doz. Dr. Friedrich Aigner, Universitätsklinik für Radiologie, Innsbruck, in „Kontrastmittelsonografie in der Uroradiologie“ auf Entwicklungen mit Anwendung der Bildfusion eingegangen, was wiederum völlig neue Bildgebungsalgorythmen öffnen zu scheint.
Es wurden die CEUS Guidelines der EFSUMB in Form der , liver and non-liver applications" angesprochen, mit dem Wunsch, dass junge Kollegen sich der Erforschung von Evidence Based appilication des CEUS in weiteren Publikationen widmen sollten, um eine langjährige Tradition der Radiologie Innsbruck weiterzuführen. Auch eine Einladung zum 39. Ländertreffen in Davos wurde ausgesprochen für Interessierte.

In der griechischen Mythologie ist der Olymp der Berg der Götter. Seinen Gipfel stellte man sich als einen lichterfüllten Ort vor, so könnte man die Methode des CEUS als eine diagnostische Lichtdurchflutung in so manchen Fraggestellungen sehen... So war der Titel dieser Veranstaltung trefflich gewählt und wurde durch beindruckendes Bildmaterial unterstrichen!

Mit besten Grüßen von der ÖGUM Tirol Andrea Klauser und Wolfgang Sturm 